\title{
Activated PTHLH Coupling Feedback Phosphoinositide to G-Protein Receptor Signal-Induced Cell Adhesion Network in Human Hepatocellular Carcinoma by Systems-Theoretic Analysis
}

\author{
Lin Wang, Juxiang Huang, ${ }^{1}$ Minghu Jiang, ${ }^{2}$ Hong Lin, ${ }^{1}$ Lianxiu Qi, ${ }^{1}$ and Haizhen Diao ${ }^{1}$ \\ ${ }^{1}$ Biomedical Center, School of Electronic Engineering, Beijing University of Posts and Telecommunications, Beijing 100876, China \\ ${ }^{2}$ Lab of Computational Linguistics, School of Humanities and Social Sciences, Tsinghua University, Beijing 100084, China \\ Correspondence should be addressed to Lin Wang, wanglin98@tsinghua.org.cn
}

Received 11 June 2012; Accepted 29 July 2012

Academic Editors: S. Guleria and S. Yasmin

Copyright () 2012 Lin Wang et al. This is an open access article distributed under the Creative Commons Attribution License, which permits unrestricted use, distribution, and reproduction in any medium, provided the original work is properly cited.

Studies were done on analysis of biological processes in the same high expression (fold change $\geq 2$ ) activated PTHLH feedback-mediated cell adhesion gene ontology (GO) network of human hepatocellular carcinoma (HCC) compared with the corresponding low expression activated GO network of no-tumor hepatitis/cirrhotic tissues (HBV or HCV infection). Activated PTHLH feedback-mediated cell adhesion network consisted of anaphase-promoting complex-dependent proteasomal ubiquitin-dependent protein catabolism, cell adhesion, cell differentiation, cell-cell signaling, G-protein-coupled receptor protein signaling pathway, intracellular transport, metabolism, phosphoinositide-mediated signaling, positive regulation of transcription, regulation of cyclin-dependent protein kinase activity, regulation of transcription, signal transduction, transcription, and transport in HCC. We proposed activated PTHLH coupling feedback phosphoinositide to G-protein receptor signal-induced cell adhesion network. Our hypothesis was verified by the different activated PTHLH feedback-mediated cell adhesion GO network of HCC compared with the corresponding inhibited GO network of no-tumor hepatitis/cirrhotic tissues, or the same compared with the corresponding inhibited GO network of HCC. Activated PTHLH coupling feedback phosphoinositide to G-protein receptor signal-induced cell adhesion network included BUB1B, GNG10, PTHR2, GNAZ, RFC4, UBE2C, NRXN3, BAP1, PVRL2, TROAP, and VCAN in HCC from GEO dataset using gene regulatory network inference method and our programming.

\section{Introduction}

PTHLH is one of our identified significant high expression (fold change $\geq 2$ ) genes in human hepatocellular carcinoma (HCC) compared with low expression no-tumor hepatitis/cirrhotic tissues (HBV or HCV infection) from GEO data set GSE10140-10141 [1].

Study of PTHLH is presented in some papers, such as Mouse pthlh gene-specific expression profiles distinguish among functional allelic variants in transfected human cancer cells [2]; parathyroid hormone-like protein alternative messenger RNA splicing pathways in human cancer cell lines [3]; parathyroid hormone-like peptide in pancreatic endocrine carcinoma and adenocarcinoma associated with hypercalcemia [4]; parathyroid hormone and parathyroid hormone-like peptide bioactivity in situ biochemistry [5]; parathyroid hormone-like protein polypeptides immunological identification and distribution in normal and malignant tissues [6]; dysregulation of parathyroid hormone-like peptide expression and secretion in a keratinocyte model of tumor progression [7]; all major lung cancer cell types produce parathyroid hormone-like protein [8]; parathyroid hormone-like peptide in normal and neoplastic mesothelial cells [9]. Yet the high expression activated PTHLH feedbackmediated cell adhesion mechanism in HCC is not clear and remains to be elucidated.

In this study, biological processes and occurrence numbers of the same activated high expression (fold change 
22) PTHLH feedback-mediated cell adhesion GO network in HCC were identified and computed compared with the corresponding low expression activated GO network of notumor hepatitis/cirrhotic tissues (HBV or HCV infection), the different compared with the corresponding inhibited PTHLH feedback-mediated cell adhesion GO network of notumor hepatitis/cirrhotic tissues, and the same compared with the corresponding inhibited GO network of HCC, respectively. Simultaneous occurrence of biological processes was identified between the same activated PTHLH feedbackmediated cell adhesion GO network of HCC (compared with the corresponding activated GO network of no-tumor hepatitis/cirrhotic tissues) and the different (compared with the corresponding inhibited PTHLH feedback-mediated cell adhesion GO network of no-tumor hepatitis/cirrhotic tissues), or the same (compared with the corresponding inhibited GO network of HCC) for putting forward hypothesis of activated PTHLH coupling feedback phosphoinositide to Gprotein receptor signal-induced cell adhesion network. Activated PTHLH feedback-mediated cell adhesion molecular network and numbers in HCC were extracted and computed from the same activated PTHLH GO-molecular network of HCC compared with the corresponding activated GOmolecular network of no-tumor hepatitis/cirrhotic tissues. PTHLH coupling feedback phosphoinositide to G-protein receptor signal-induced cell adhesion molecular relationship in HCC was identified including different molecules but same GO term and same molecule but different GO terms from the same activated PTHLH GO-molecular network of HCC compared with the corresponding activated GOmolecular network of no-tumor hepatitis/cirrhotic tissues.

\section{Materials and Methods}

Microarray 6,144 genes were used for analyzing activated PTHLH feedback-mediated cell adhesion mechanism of HCC based on GEO data set GSE10140-10141 (http://www .ncbi.nlm.nih.gov/geo/query/acc.cgi?acc=GSE10140, http:// www.ncbi.nlm.nih.gov/geo/query/acc.cgi?acc=GSE10141).

The raw microarray data was preprocessed by log base 2 .

225 significant high expression (fold change $\geq 2$ ) molecules in HCC compared with no-tumor hepatitis/cirrhotic tissues (HBV or HCV infection) were identified using significant analysis of microarrays (SAM) (http://www-stat .stanford.edu/ tibs/SAM/) [10]. We selected two classes paired and minimum fold change $\geq 2$ under the falsediscovery rate was $0 \%$.

Activated PTHLH feedback-mediated cell adhesion mechanism of HCC was analyzed by using Molecule Annotation System, MAS (CapitalBio Corporation, Beijing, China; http://bioinfo.capitalbio.com/mas3/). The primary databases of MAS integrated various well-known biological resources, such as Gene Ontology (http://www.geneontology.org/), KEGG (http://www.genome.jp/kegg/), BioCarta (http://www .biocarta.com/), GenMapp (http://www.genmapp.org/), HPRD (http://www.hprd.org/), MINT (http://mint.bio.uniroma2.it/mint/Welcome.do), BIND (http://www.blueprint .org/), Intact (http://www.ebi.ac.uk/intact/), UniGene (http://www.ncbi.nlm.nih.gov/unigene), OMIM (http:// www.ncbi.nlm.nih.gov/entrez/query.fcgi? $\mathrm{db}=\mathrm{OMIM}$ ), and disease (http://bioinfo.capitalbio.com/mas3/).

Biological processes and occurrence numbers of the same activated high expression (fold change $\geq 2$ ) PTHLH feedback-mediated cell adhesion GO network in HCC were identified and computed compared with the corresponding low expression activated GO network of notumor hepatitis/cirrhotic tissues (HBV or HCV infection), the different compared with the corresponding inhibited PTHLH feedback-mediated cell adhesion GO network of notumor hepatitis/cirrhotic tissues, and the same compared with the corresponding inhibited GO network of HCC by our programming, respectively.

Simultaneous occurrence of biological processes was identified between the same activated PTHLH feedbackmediated cell adhesion GO network of HCC (compared with the corresponding activated GO network of no-tumor hepatitis/cirrhotic tissues) and the different (compared with the corresponding inhibited PTHLH feedback-mediated cell adhesion GO network of no-tumor hepatitis/cirrhotic tissues), or the same (compared with the corresponding inhibited GO network of HCC) for putting forward hypothesis of activated PTHLH coupling feedback phosphoinositide to G-protein receptor signal-induced cell adhesion network by our programming, respectively.

Activated PTHLH feedback-mediated cell adhesion molecular network and numbers in HCC were extracted and computed from the same activated PTHLH GO-molecular network of HCC compared with the corresponding activated GO-molecular network of no-tumor hepatitis/cirrhotic tissues by our programming, respectively.

At last, PTHLH coupling feedback phosphoinositide to G-protein receptor signal-induced cell adhesion molecular relationship in HCC was identified including different molecules but same GO term and same molecule but different GO terms from the same activated PTHLH GOmolecular network of HCC compared with the corresponding activated GO-molecular network of no-tumor hepatitis/cirrhotic tissues, and constructed network by GRNInfer [11] and our articles [12-25] and illustrated by GVedit tool.

\section{Results}

Biological processes and occurrence numbers of the same activated high expression (fold change $\geq 2$ ) PTHLH feedback-mediated cell adhesion GO network in HCC were identified and computed compared with the corresponding low expression activated GO network of no-tumor hepatitis/cirrhotic tissues (HBV or HCV infection), the different compared with the corresponding inhibited PTHLH feedback-mediated cell adhesion GO network of no-tumor hepatitis/cirrhotic tissues, and the same compared with the corresponding inhibited GO network of HCC, respectively.

The same biological processes of activated PTHLH feedback-mediated cell adhesion GO network in HCC included anaphase-promoting complex-dependent proteasomal ubiquitin-dependent protein catabolism, cell adhesion, cell differentiation, cell-cell signaling, endothelial 
cell migration, G-protein-coupled receptor protein signaling pathway, G-protein signaling, intracellular transport, metabolism, phosphoinositide-mediated signaling, positive regulation of transcription, protein amino acid phosphorylation, regulation of cyclin-dependent protein kinase activity, regulation of transcription, signal transduction, transcription, and transport compared with the corresponding activated GO network of no-tumor hepatitis/cirrhotic tissues.

The different biological processes of activated PTHLH feedback-mediated cell adhesion GO network in HCC contained integrin-mediated signaling pathway, intracellular transport, microtubule cytoskeleton organization and biogenesis, regulation of cell growth, regulation of cyclindependent protein kinase activity compared with the corresponding inhibited GO network of no-tumor hepatitis/cirrhotic tissues.

The same biological processes of activated PTHLH feedback-mediated cell adhesion GO network in HCC included anaphase-promoting complex-dependent proteasomal ubiquitin-dependent protein catabolism, cell adhesion, cell differentiation, cell-cell signaling, DNA repair, G-protein-coupled receptor protein signaling pathway, integrin-mediated signaling pathway, metabolism, nucleotide and nucleic acid metabolism, oxidation reduction, phosphoinositide-mediated signaling, positive regulation of transcription, protein modification, proteolysis, regulation of cyclin-dependent protein kinase activity, regulation of transcription, signal transduction, and transcription, transport compared with the corresponding inhibited GO network of HCC, as shown in Table 1.

Activated PTHLH feedback-mediated cell adhesion molecular network and numbers in HCC were extracted and computed from the same activated PTHLH GO-molecular network of HCC compared with the corresponding activated GO-molecular network of no-tumor hepatitis/cirrhotic tissues. Our result showed that PTHLH feedback-mediated cell adhesion molecular network consisted of BUB1B, GNG10, PTHR2, GNAZ, RFC4, UBE2C, NRXN3, BAP1, PVRL2, TROAP, VCAN, CCNA2, CDC6, CDKN2C, and ENAH in HCC, as shown in Table 2.

\section{Discussion}

Our aim is to study novel high expression-activated PTHLH feedback-mediated cell adhesion mechanism in HCC. In this study, biological processes and occurrence numbers of the same activated high expression (fold change $\geq 2$ ) PTHLH feedback-mediated cell adhesion GO network in HCC were identified and computed compared with the corresponding low expression activated GO network of notumor hepatitis/cirrhotic tissues (HBV or HCV infection), the different compared with the corresponding inhibited PTHLH feedback-mediated cell adhesion GO network of notumor hepatitis/cirrhotic tissues, and the same compared with the corresponding inhibited GO network of HCC, respectively (Table 1).

Simultaneous occurrence of biological processes was identified between the same activated PTHLH feedbackmediated cell adhesion GO network of HCC (compared with the corresponding activated GO network of no-tumor hepatitis/cirrhotic tissues) and the different (compared with the corresponding inhibited PTHLH feedback-mediated cell adhesion GO network of no-tumor hepatitis/cirrhotic tissues), or the same (compared with the corresponding inhibited GO network of HCC) for putting forward hypothesis of activated PTHLH coupling feedback phosphoinositide to G-protein receptor signal-induced cell adhesion network, respectively.

Simultaneous occurrence of biological processes consisted of intracellular transport, regulation of cyclindependent protein kinase activity between the same activated PTHLH feedback-mediated cell adhesion GO network of HCC (compared with the corresponding activated GO network of no-tumor hepatitis/cirrhotic tissues) and the different (compared with the corresponding inhibited PTHLH feedback-mediated cell adhesion GO network of no-tumor hepatitis/cirrhotic tissues).

Simultaneous occurrence of biological processes included anaphase-promoting complex-dependent proteasomal ubiquitin-dependent protein catabolism, cell adhesion, cell differentiation, cell-cell signaling, G-proteincoupled receptor protein signaling pathway, metabolism, phosphoinositide-mediated signaling, positive regulation of transcription, regulation of cyclin-dependent protein kinase activity, regulation of transcription, signal transduction, transcription, transport between the same activated PTHLH feedback-mediated cell adhesion GO network of HCC (compared with the corresponding activated GO network of notumor hepatitis/cirrhotic tissues), and the same (compared with the corresponding inhibited GO network of HCC).

The studies of phosphoinositide with adhesion are presented as follows. Phosphoinositide lipid phosphatase SHIP1 and PTEN coordinate to regulate cell migration and adhesion [26], TAPP2 links phosphoinositide 3-kinase signaling to Bcell adhesion through interaction with the cytoskeletal protein utrophin: expression of a novel cell adhesion-promoting complex in B-cell leukemia [27], neuregulin-1 regulates cell adhesion via an ErbB2/phosphoinositide-3 kinase/Aktdependent pathway: potential implications for schizophrenia and cancer [28], stromal cell-derived factor-1alpha stimulates tyrosine phosphorylation of multiple focal adhesion proteins and induces migration of hematopoietic progenitor cells: roles of phosphoinositide-3 kinase and protein kinase C [29], and functional association of platelet endothelial cell adhesion molecule-1 and phosphoinositide 3-kinase in human neutrophils [30]. Therefore, we proposed activated PTHLH coupling feedback phosphoinositide to G-protein receptor signal-induced cell adhesion network in HCC.

Activated PTHLH feedback-mediated cell adhesion molecular network and numbers in HCC were extracted and computed from the same activated PTHLH GO-molecular network of HCC compared with the corresponding activated GO-molecular network of no-tumor hepatitis/cirrhotic tissues (Table 2). PTHLH coupling feedback phosphoinositide to G-protein receptor signal-induced cell adhesion molecular relationship in HCC was identified including different molecules but same GO term and same molecule 
TABLe 1: (a) Biological processes and occurrence numbers of the same activated high expression (fold change $\geq 2$ ) PTHLH feedbackmediated cell adhesion GO network in HCC compared with the corresponding low expression activated GO network of no-tumor hepatitis/cirrhotic tissues (HBV or HCV infection), (b) the different compared with the corresponding inhibited PTHLH feedback-mediated cell adhesion GO network of no-tumor hepatitis/cirrhotic tissues, and (c) the same compared with the corresponding inhibited GO network of HCC by our programming.

(a) Biological process and occurrence number of GO term

Anaphase-promoting complex-dependent proteasomal ubiquitin-dependent protein catabolism

Cell adhesion

Cell differentiation

Cell-cell signaling

Endothelial cell migration

G-protein-coupled receptor protein signaling pathway

G-protein signaling

Intracellular transport

metabolism

Phosphoinositide-mediated signaling

Positive regulation of transcription

Protein amino acid phosphorylation

Regulation of cyclin-dependent protein kinase activity

Transport

(b) Biological process and occurrence number of GO term

Integrin-mediated signaling pathway

Intracellular transport

Microtubule cytoskeleton organization and biogenesis

Regulation of cell growth

Regulation of cyclin-dependent protein kinase activity

(c) Biological process and occurrence number of GO term

Anaphase-promoting complex-dependent proteasomal ubiquitin-dependent protein catabolism

Cell adhesion

Cell differentiation

Cell-cell signaling

DNA repair

G-protein-coupled receptor protein signaling pathway

Integrin-mediated signaling pathway

Metabolism

Nucleotide and nucleic acid metabolism

Oxidation reduction

Phosphoinositide-mediated signaling

Positive regulation of transcription

Protein modification

Proteolysis

Regulation of cyclin-dependent protein kinase activity

Regulation of transcription 
TABLE 2: Activated PTHLH feedback-mediated cell adhesion molecular network and numbers in HCC from the same activated PTHLH GO-molecular network of HCC compared with the corresponding activated GO-molecular network of no-tumor hepatitis/cirrhotic tissues by our programming.

\begin{tabular}{ll}
\hline Molecular name and number & \\
\hline BUB1B, GNG10, PTHR2, GNAZ, RFC4, UBE2C, NRXN3, BAP1, PVRL2, TROAP, VCAN, CCNA2, CDC6, CDKN2C, ENAH & 15 \\
\hline
\end{tabular}

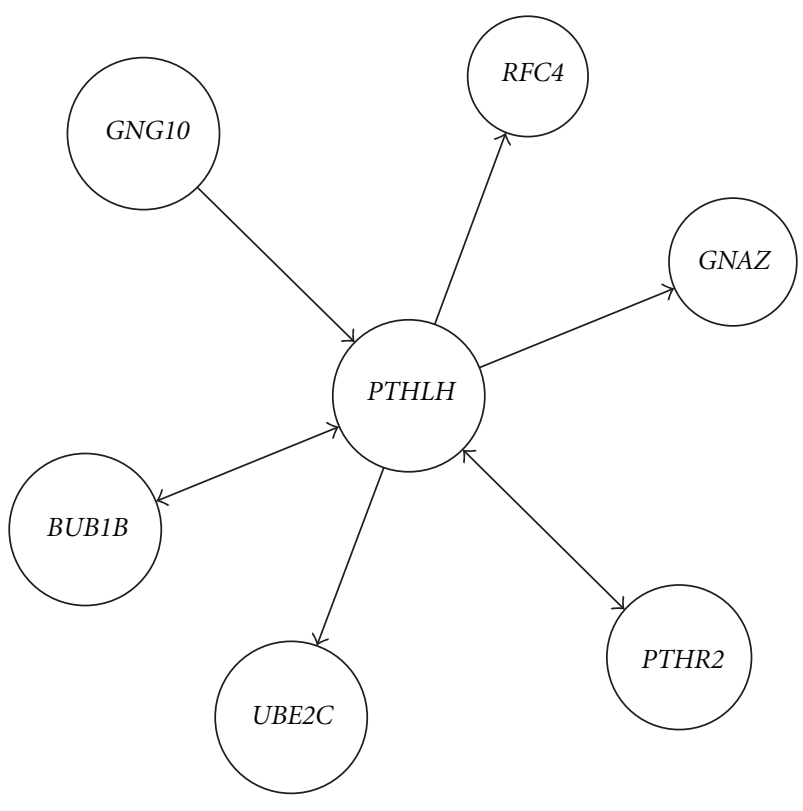

Figure 1: Activated PTHLH coupling feedback phosphoinositide to G-protein receptor signal network construction including different molecules but same GO term and same molecule but different GO terms in HCC from the same activated PTHLH GO-molecular network of HCC compared with the corresponding activated GO-molecular network of no-tumor hepatitis/cirrhotic tissues by GRNInfer and our programming.

but different GO terms from the same activated PTHLH GO-molecular network of HCC compared with the corresponding activated GO-molecular network of no-tumor hepatitis/cirrhotic tissues. Activated PTHLH coupling feedback phosphoinositide to G-protein receptor signal network included BUB1B, GNG10, PTHR2, GNAZ, PTHR2, BUB1B, RFC4, and UBE2C and activated PTHLH feedback cell adhesion network NRXN3, BAP1, NRXN3, PVRL2, TROAP, and VCAN in HCC, as shown in Figures 1 and 2.

In summary, studies were done on analysis of biological processes in the same high expression (fold change $\geq 2$ ) activated PTHLH feedback-mediated cell adhesion GO network of HCC compared with the corresponding low expression activated GO network of no-tumor hepatitis/cirrhotic tissues (HBV or HCV infection). Activated PTHLH feedback-mediated cell adhesion network consisted of anaphase-promoting complex-dependent proteasomal ubiquitin-dependent protein catabolism, cell adhesion, cell differentiation, cell-cell signaling, G-protein-coupled receptor protein signaling pathway, intracellular transport, metabolism, phosphoinositide-mediated signaling, positive

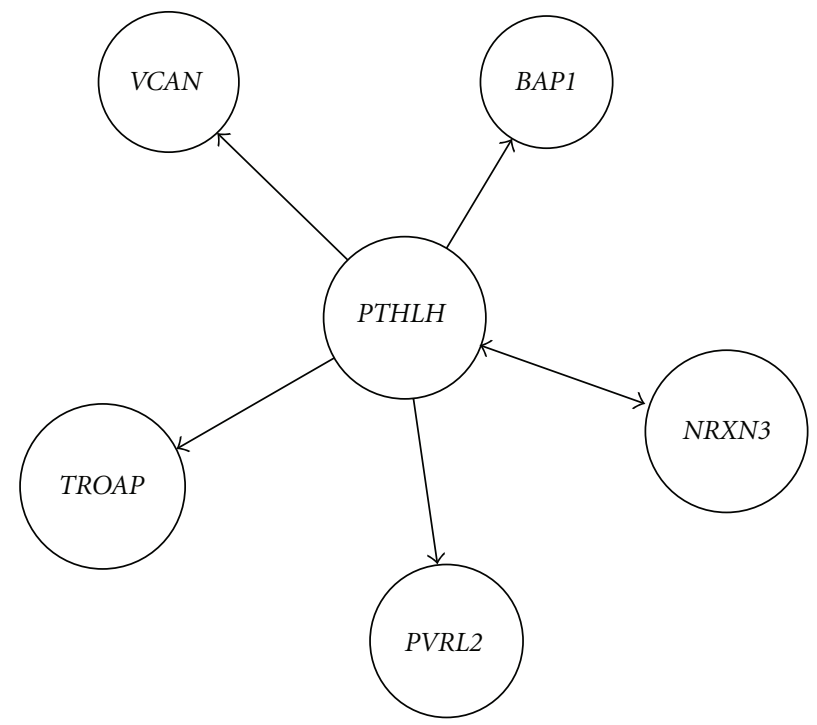

Figure 2: Activated PTHLH feedback cell adhesion network construction including different molecules but same GO term and same molecule but different GO terms in HCC from the same activated PTHLH GO-molecular network of HCC compared with the corresponding activated GO-molecular network of no-tumor hepatitis/cirrhotic tissues by GRNInfer and our programming.

regulation of transcription, regulation of cyclin-dependent protein kinase activity, regulation of transcription, signal transduction, transcription, and transport in HCC. We proposed activated PTHLH coupling feedback phosphoinositide to G-protein receptor signal-induced cell adhesion network. Our hypothesis was verified by the different activated PTHLH feedback-mediated cell adhesion GO network of HCC compared with the corresponding inhibited GO network of no-tumor hepatitis/cirrhotic tissues, or the same compared with the corresponding inhibited GO network of HCC. Activated PTHLH coupling feedback phosphoinositide to G-protein receptor signal-induced cell adhesion network included BUB1B, GNG10, PTHR2, GNAZ, RFC4, UBE2C, NRXN3, BAP1, PVRL2, TROAP, and VCAN in HCC from GEO data set using gene regulatory network inference method and our programming.

\section{Authors' Contribution}

Equal contribution.

\section{Acknowledgments}

This work was supported by the National Natural Science Foundation of China (no. 61171114), the Returned 
Overseas Chinese Scholars for Scientific research Foundation of State Education Ministry, Significant Science and Technology Project for New Transgenic Biological Species (2009ZX08012-001B), Automatical Scientific Planning of Tsinghua University (20111081023 and 20111081010), and State Key Lab of Pattern Recognition Open Foundation.

\section{References}

[1] Y. Hoshida, A. Villanueva, M. Kobayashi et al., "Gene expression in fixed tissues and outcome in hepatocellular carcinoma," The New England Journal of Medicine, vol. 359, no. 19, pp. 1995-2004, 2008.

[2] R. Gianni-Barrera, M. Gariboldi, L. De Cecco, G. Manenti, and T. A. Dragani, "Specific gene expression profiles distinguish among functional allelic variants of the mouse Pthlh gene in transfected human cancer cells," Oncogene, vol. 25, no. 32, pp. 4501-4504, 2006.

[3] D. W. Brandt, W. Wachsman, and L. J. Deftos, "Parathyroid hormone-like protein: alternative messenger RNA splicing pathways in human cancer cell lines," Cancer Research, vol. 54, no. 3, pp. 850-853, 1994.

[4] B. A. Miraliakbari, S. L. Asa, and S. F. Boudreau, "Parathyroid hormone-like peptide in pancreatic endocrine carcinoma and adenocarcinoma associated with hypercalcemia," Human Pathology, vol. 23, no. 8, pp. 884-887, 1992.

[5] N. Loveridge, V. Dean, D. Goltzman, and G. N. Hendy, "Bioactivity of parathyroid hormone and parathyroid hormone-like peptide: agonist and antagonist activities of amino-terminal fragments as assessed by the cytochemical bioassay and in situ biochemistry," Endocrinology, vol. 128, no. 4, pp. 1938-1946, 1991.

[6] S. Kramer, F. H. Reynolds, M. Castillo, D. M. Valenzuela, M. Thorikay, and J. M. Sorvillo, "Immunological identification and distribution of parathyroid hormone-like protein polypeptides in normal and malignant tissues," Endocrinology, vol. 128, no. 4, pp. 1927-1937, 1991.

[7] J. Henderson, M. Sebag, J. Rhim, D. Goltzman, and R. Kremer, "Dysregulation of parathyroid hormone-like peptide expression and secretion in a keratinocyte model of tumor progression," Cancer Research, vol. 51, no. 24, pp. 6521-6528, 1991.

[8] D. W. Brandt, D. W. Burton, A. F. Gazdar, H. E. Oie, and L. J. Deftos, "All major lung cancer cell types produce parathyroid hormone-like protein: heterogeneity assessed by high performance liquid chromatography," Endocrinology, vol. 129, no. 5, pp. 2466-2470, 1991.

[9] P. McAuley, S. L. Asa, B. Chiu, J. Henderson, D. Goltzman, and D. J. Drucker, "Parathyroid hormone-like peptide in normal and neoplastic mesothelial cells," Cancer, vol. 66, no. 9, pp. 1975-1979, 1990.

[10] J. D. Storey, “A direct approach to false discovery rates," Journal of the Royal Statistical Society B, vol. 64, no. 3, pp. 479-498, 2002.

[11] Y. Wang, T. Joshi, X. S. Zhang, D. Xu, and L. Chen, "Inferring gene regulatory networks from multiple microarray datasets," Bioinformatics, vol. 22, no. 19, pp. 2413-2420, 2006.

[12] L. Wang, Y. Sun, M. Jiang, and X. Zheng, "Integrative decomposition procedure and kappa statistics for the distinguished single molecular network construction and analysis," Journal of Biomedicine and Biotechnology, vol. 2009, Article ID 726728, 6 pages, 2009.
[13] L. Wang, Y. Sun, M. Jiang, S. Zhang, and S. Wolfl, "FOS proliferating network construction in early colorectal cancer (CRC) based on integrative significant function cluster and inferring analysis," Cancer Investigation, vol. 27, no. 8, pp. 816-824, 2009.

[14] L. Wang, L. Sun, J. Huang, and M. Jiang, "Cyclin-dependent kinase inhibitor 3 (CDKN3) novel cell cycle computational network between human non-malignancy associated hepatitis/cirrhosis and hepatocellular carcinoma (HCC) transformation," Cell Proliferation, vol. 44, no. 3, pp. 291-299, 2011.

[15] L. Wang, J. Huang, M. Jiang, and X. Zheng, "AFP computational secreted network construction and analysis between human hepatocellular carcinoma (HCC) and no-tumor hepatitis/cirrhotic liver tissues," Tumour Biology, vol. 31, no. 5, pp. 417-425, 2010.

[16] L. Wang, J. Huang, M. Jiang, and L. Sun, "MYBPC1 computational phosphoprotein network construction and analysis between frontal cortex of HIV encephalitis (HIVE) and HIVEcontrol patients," Cellular and Molecular Neurobiology, vol. 31, no. 2, pp. 233-241, 2011.

[17] L. Wang, J. Huang, M. Jiang, and L. Sun, "Survivin (BIRC5) cell cycle computational network in human no-tumor hepatitis/cirrhosis and hepatocellular carcinoma transformation," Journal of Cellular Biochemistry, vol. 112, no. 5, pp. 1286-1294, 2011.

[18] L. Wang, J. Huang, M. Jiang, and H. Lin, "Signal transducer and activator of transcription 2 (STAT2) metabolism coupling postmitotic outgrowth to visual and sound perception network in human left cerebrum by biocomputation," Journal of molecular neuroscience, vol. 47, no. 3, pp. 649-58, 2012.

[19] L. Wang, J. Huang, and M. Jiang, "RRM2 computational phosphoprotein network construction and analysis between notumor hepatitis/cirrhotic liver tissues and human hepatocellular carcinoma (HCC)," Cellular Physiology and Biochemistry, vol. 26, no. 3, pp. 303-310, 2010.

[20] L. Wang, J. Huang, and M. Jiang, "CREB5 computational regulation network construction and analysis between frontal cortex of HIV encephalitis (HIVE) and HIVE-control patients," Cell Biochemistry and Biophysics, vol. 60, no. 3, pp. 199-207, 2011.

[21] Y. Sun, L. Wang, and L. Liu, "Integrative decomposition procedure and Kappa statistics set up ATF2 ion binding module in malignant pleural mesothelioma (MPM)," Frontiers of Electrical and Electronic Engineering in China, vol. 3, no. 4, pp. 381-387, 2008.

[22] Y. Sun, L. Wang, M. Jiang, J. Huang, Z. Liu, and S. Wolfl, "Secreted phosphoprotein 1 upstream invasive network construction and analysis of lung adenocarcinoma compared with human normal adjacent tissues by integrative biocomputation," Cell Biochemistry and Biophysics, vol. 56, no. 2, pp. 5971, 2010.

[23] L. Sun, L. Wang, M. Jiang, J. Huang, and H. Lin, "Glycogen debranching enzyme 6 (AGL), enolase 1 (ENOSF1), ectonucleotide pyrophosphatase 2 (ENPP2_1), glutathione S-transferase 3 (GSTM3_3) and mannosidase (MAN2B2) metabolism computational network analysis between chimpanzee and human left cerebrum," Cell Biochemistry and Biophysics, vol. 61, no. 3, pp. 493-505, 2011.

[24] J. X. Huang, L. Wang, and M. H. Jiang, "TNFRSF11B computational development network construction and analysis between frontal cortex of HIV encephalitis (HIVE) and HIVEcontrol patients," Journal of Inflammation, vol. 7, article 50, 2010. 
[25] J. Huang, L. Wang, M. Jiang, and X. Zheng, "Interferon $\alpha$ inducible protein 27 computational network construction and comparison between the frontal cortex of HIV encephalitis (HIVE) and HIVE-control patients," Open Genomics Journal, vol. 3, no. 1, pp. 1-8, 2010.

[26] S. Mondal, K. K. Subramanian, J. Sakai, B. Bajrami, and H. R. Luo, "Phosphoinositide lipid phosphatase SHIP1 and PTEN coordinate to regulate cell migration and adhesion," Molecular Biology of the Cell, vol. 23, no. 7, pp. 1219-1230, 2012.

[27] J. L. Costantini, S. M. S. Cheung, S. Hou et al., "TAPP2 links phosphoinositide 3-kinase signaling to B-cell adhesion through interaction with the cytoskeletal protein utrophin: expression of a novel cell adhesion-promoting complex in Bcell leukemia," Blood, vol. 114, no. 21, pp. 4703-4712, 2009.

[28] C. G. Kanakry, Z. Li, Y. Nakai, Y. Sei, and D. R. Weinberger, "Neuregulin-1 regulates cell adhesion via an ErbB2/phosphoinositide-3 kinase/akt-dependent pathway: potential implications for schizoprenia and cancer," PLoS ONE, vol. 2, no. 12, Article ID e1369, 2007.

[29] J. F. Wang, I. W. Park, and J. E. Groopman, "Stromal cell-derived factor- $1 \alpha$ stimulates tyrosine phosphorylation of multiple focal adhesion proteins and induces migration of hematopoietic progenitor cells: roles of phosphoinositide-3 kinase and protein kinase C," Blood, vol. 95, no. 8, pp. 25052513, 2000.

[30] F. Pellegatta, S. L. Chierchia, and M. R. Zocchi, "Functional association of platelet endothelial cell adhesion molecule-1 and phosphoinositide 3-kinase in human neutrophils," Journal of Biological Chemistry, vol. 273, no. 43, pp. 27768-27771, 1998. 

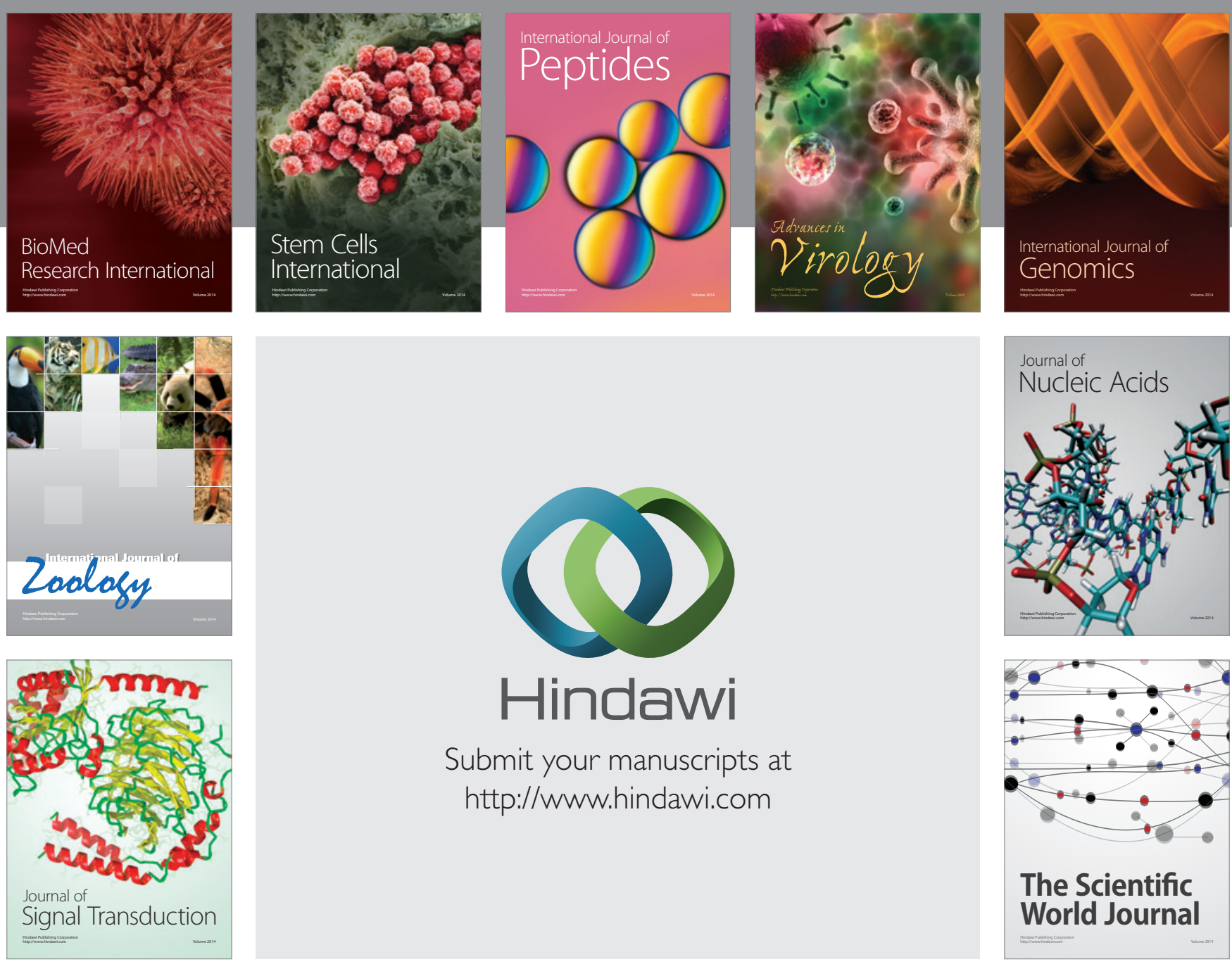

Submit your manuscripts at

http://www.hindawi.com
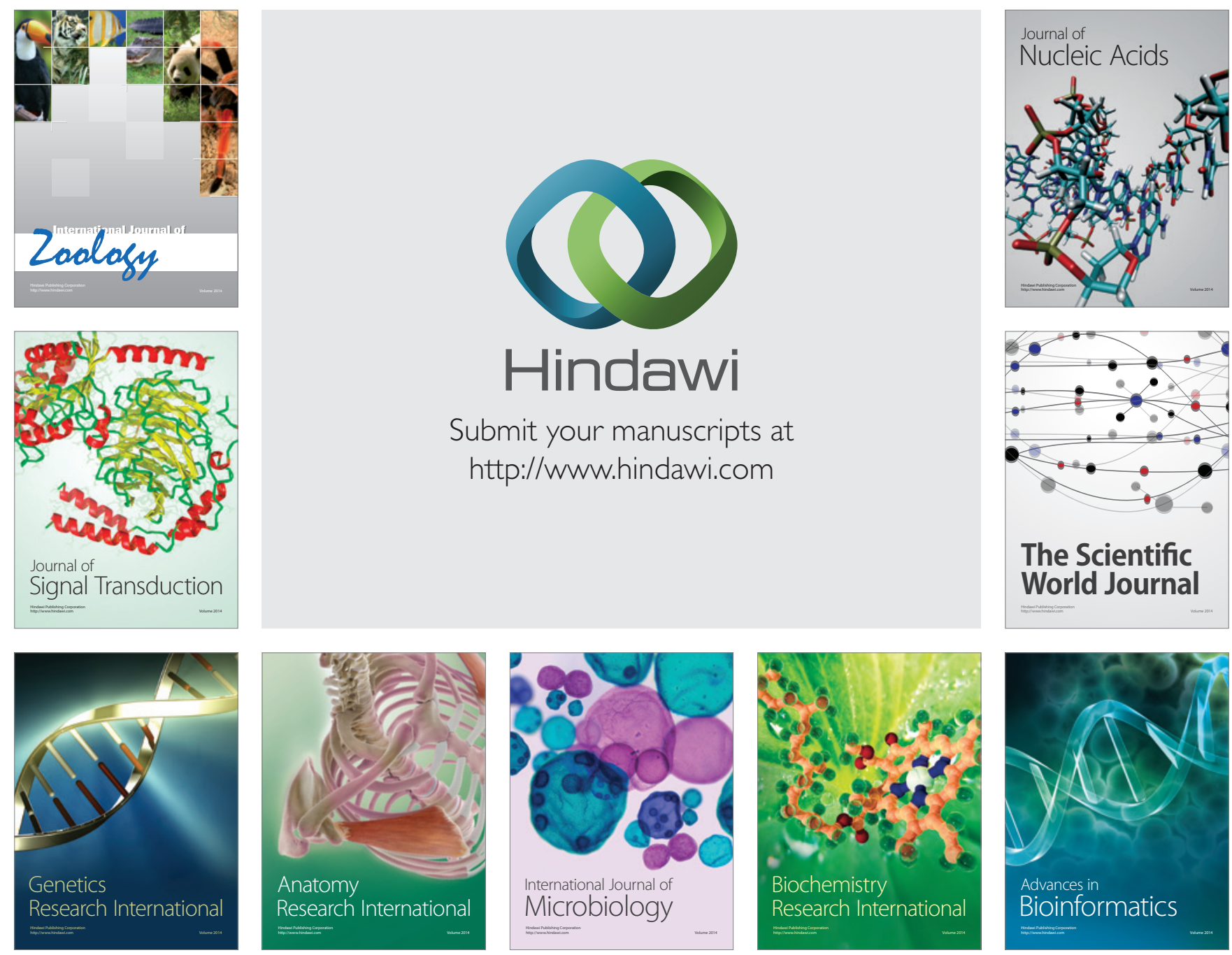

The Scientific World Journal
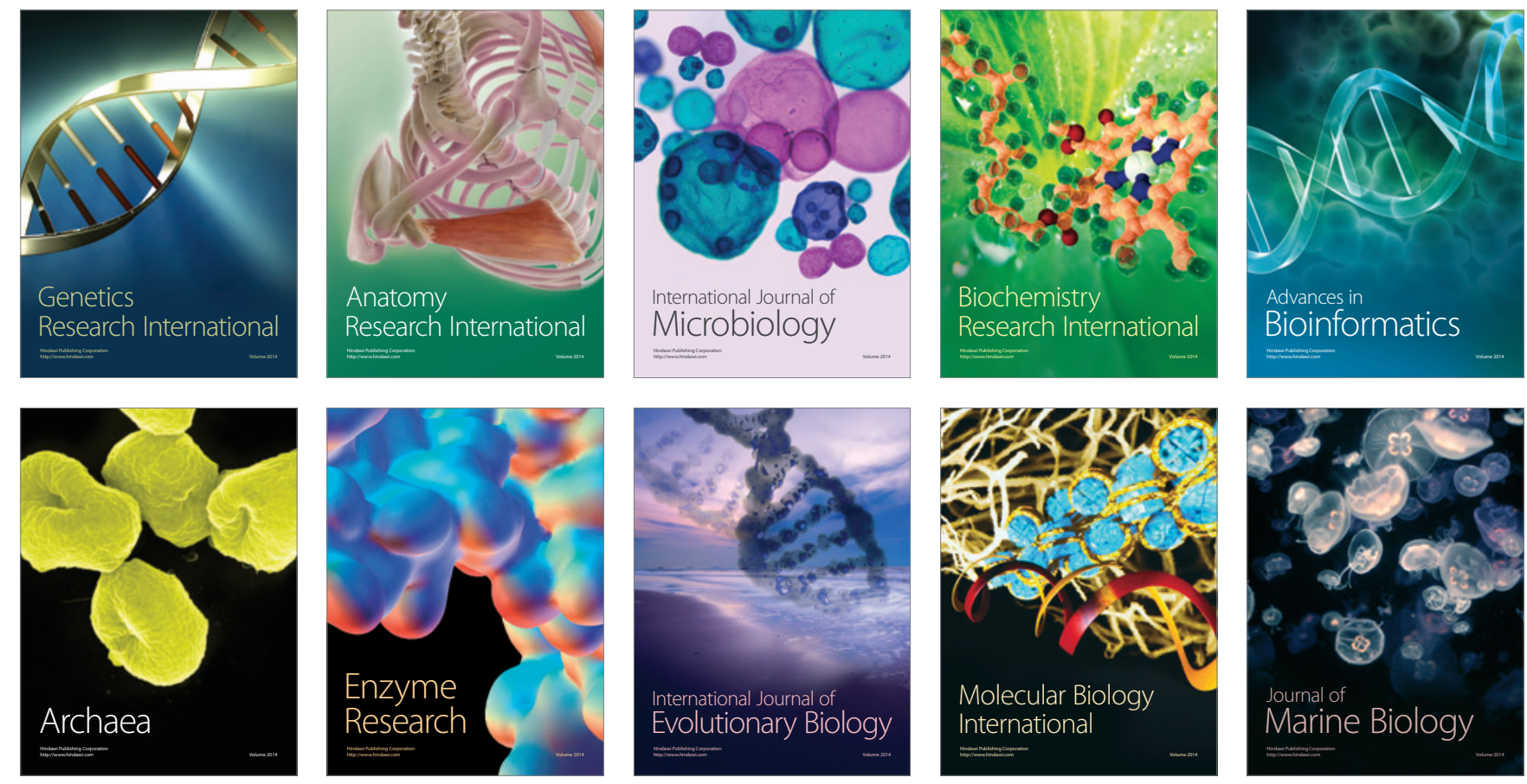